Journal of Zhejiang University-SCIENCE A (Applied Physics \& Engineering)

ISSN 1673-565X (Print); ISSN 1862-1775 (Online)

www.zju.edu.cn/jzus; www.springerlink.com

E-mail: jzus@zju.edu.cn

\title{
Squeal noise in hydraulic poppet valves*
}

\author{
Da-yun YI, Liang LU ${ }^{\dagger *}$, Jun ZOU, Xin FU \\ (The State Key Laboratory of Fluid Power and Mechatronic Systems, Zhejiang University, Hangzhou 310027, China) \\ "E-mail: luliang829@zju.edu.cn \\ Received Nov. 25, 2014; Revision accepted Oct. 19, 2015; Crosschecked Mar. 15, 2016
}

\begin{abstract}
The poppet valve is a fundamental component in fluid power systems. Under particular conditions, annoying "squeal" noises may be generated in hydraulic poppet valves. In the present study, the frequency spectrum of the squeal noise is obtained by analyzing the sampling data from the accelerometer mounted on the valve body. It is found that the flow velocity, pressure, and structural parameters have crucial effects on the properties of squeal noise, especially frequency. Larger valve chamber volume or lower backpressure leads to lower fundamental frequency of the squeal noise. An explanation for the squeal noise, as a result of Helmholtz resonance, is suggested and proved by experimental results.
\end{abstract}

Key words: Poppet valve, Squeal noise, Helmholtz resonance, Fluid acoustics, Shear layer instability http://dx.doi.org/10.1631/jzus.A1400351

CLC number: TH137.52

\section{Introduction}

In fluid power systems, the relief valve is usually used to hold the system pressure at the set value by discharging excess fluid to the reservoir. To meet the requirements of large area gradient and high flow capacity, the poppet structure is adopted in almost all relief valves. In addition to the instability of the relief valve, the noise is also the focus of the customers and designers.

The noises in the poppet valve have two main sources, including mechanical noise and flowinduced noise. The mechanical noise, arising from the motion of valve components, is characterized by low frequency. It can be suppressed by designing a valve with good stability, for instance, selecting a high-stiffness spring or adding a small orifice at the

\footnotetext{
${ }^{\ddagger}$ Corresponding author

* Project supported by the National Natural Science Foundation of China (No. 51475415), the Science Fund for Creative Research Groups of the National Natural Science Foundation of China (No. 51221004), and the Fundamental Research Funds for the Central Universities, China

(D) ORCID: Da-yun YI, http://orcid.org/0000-0001-9274-6632; Liang LU, http://orcid.org/0000-0002-9403-330X

(C) Zhejiang University and Springer-Verlag Berlin Heidelberg 2016
}

upstream end of the poppet (Shin, 1991). Flowinduced noises almost originate from the turbulent flow during phase change. One of them, namely the "squeal" noise, can be heard occasionally under particular conditions during its operation and is characterized by a harsh tone. Unlike cavitation noises of a large bandwidth (Lu et al., 2012), the squeal noise has a monotone tone, to which human beings are more sensitive and which is intolerable.

The squeal noise is common in air control applications because shock waves are easily stimulated by a high-speed air jet (Raman, 1999). The instability of the shear layer is responsible for the squeal noise in air valves (Testud et al., 2009). In order to study the instability of the shear flow, Balatka et al. (1996) investigated the flow behavior in an annular conical passage when the flow undergoes transition from a considerably stable laminar flow to a selfsustained oscillating flow. As for homogeneous and density-stratified inviscid shear flows, Baines and Mitsudera (1994) found that the instability of the free-shear layer is caused by the wave interactions rather than the Kelvin-Helmholtz instability.

Up to now, few reports are available on the squeal noise in hydraulic valves. Porteiro et al. (1997) 
and Rahman et al. (1997) proposed that the instability of the shear layer induces the squeal noise in a counterbalance valve. It is well known that the vortex caused by the instability of the shear layer in the flow passage includes complex frequency contents. In fact, only some signals with a particular frequency are amplified as the squeal noise heard in the poppet valve during its operation.

In the present study, the authors intend to reveal the mechanism of selective amplification of the fluid-induced noise in the poppet valves at particular frequencies. First, vibrating signals were acquired under various pressure and geometric conditions. Then, an assumption of squeal noise based on Helmholtz resonance was made and verified by experimental and analytical analyses.

\section{Experimental}

The test bench, shown in Fig. 1, comprises a variable displacement piston pump, a relief valve, a test valve, a throttling valve, a shutoff valve, and associated measuring apparatuses. The flowmeter records the volume of oil passing through the test poppet valve per minute. The throttling valve at the exit of the test valve is used to hold back pressure. The pressure gauges show the pressure at the inlet and the outlet, respectively. The miniature highresponse pressure sensor is used to detect the pressure fluctuation in the valve chamber as the squeal noise is generated. A piezoelectric accelerometer 4517 (Brüel \& Kjær, Danmark) fixed on the surface of the valve body is adopted to detect the acceleration signals, and its frequency response ranges from $1 \mathrm{~Hz}$ to $20 \mathrm{kHz}$. This instrument is usually used to study the frequency spectra of fluid-induced noise (Nagaya and Murase, 2012) rather than the hydrophone because of the effect of the acoustic reflection on the narrow flow passage. The opening of the test poppet valve is measured by a micrometer. The flow behavior in the test valve is recorded by a high-speed camera with the assistance of desired illumination.

The valve body of the test valve, made of transparent Plexiglas, is mounted on the test bench. The poppet with a screw rod is fastened tightly by a nut. The small metering orifice at the upstream end of the valve seat is used to produce a pressure drop, which is widely applied in commercial products. Valve bodies with different lengths of the downstream valve chamber were tested during the experiments. The detailed sizes are listed in Table 1.

When the oil fluid (VG46) passes through the narrow passage formed by the poppet and the valve seat, the oscillating flow in the annular conical passage caused by the instability of the shear layer can be characterized by the Strouhal number $(S t)$, which is expressed as follows (Nakano et al., 1988):

$$
S t=f_{\mathrm{s}} \cdot \frac{L_{\mathrm{c}}}{U}
$$

where $f_{\mathrm{s}}$ is the shedding frequency of the vortex induced by the instability of the shear layer, $L_{\mathrm{c}}$ is the characteristic length, and $U$ is the mean flow velocity.

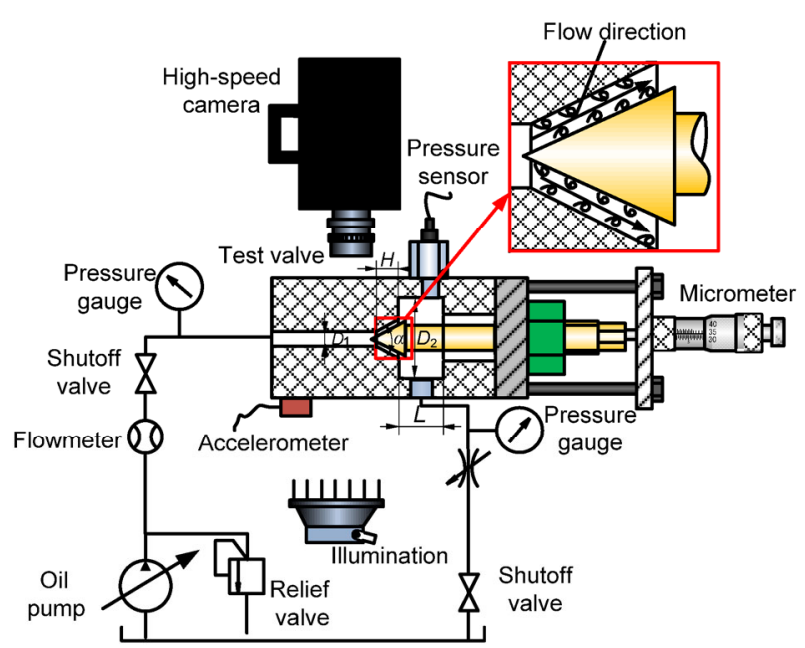

Fig. 1 Schematic diagram of the test bench

Table 1 Structural parameters of test valves

\begin{tabular}{cccccc}
\hline Type & $D_{1}(\mathrm{~mm})$ & $D_{2}(\mathrm{~mm})$ & $L(\mathrm{~mm})$ & $\alpha\left(^{\circ}\right)$ & $H(\mathrm{~mm})$ \\
\hline V01 & 4 & 28 & 12 & 60 & 5 \\
V02 & 4 & 28 & 24 & 60 & 5 \\
\hline
\end{tabular}

$D_{1}$ and $D_{2}$ are the diameters of orifice and valve chamber, respectively, $L$ is the length of valve chamber, $\alpha$ is the cone angle, and $H$ is the height of valve seat

\section{Results and discussion}

\subsection{Spectral characteristics of squeal noise}

Fig. 2 shows the pressure fluctuations in the downstream valve chamber as the squeal noise 
occurs in the test valve. The pressure fluctuates critically in the valve chamber. Some periodic properties can be observed from the pressure signals. In order to give a deep insight into the characteristics of squeal noise, spectrum analysis is applied. The spectral content of squeal noise can be obtained by analyzing the sampling data from the accelerometer via fast Fourier transform techniques. Fig. 3 shows the typical spectrum of the squeal noise generated in the test valve. The spectrum is characterized by uniformly spaced peaks with a sharp profile and high frequency. The fundamental frequency $f_{1}$ is $3342 \mathrm{~Hz}$ $\left(f_{1} \approx 1 / \Delta t\right)$, and harmonics occur at $6684 \mathrm{~Hz}$ and $10026 \mathrm{~Hz}$, which are the integer multiples of the fundamental frequency.

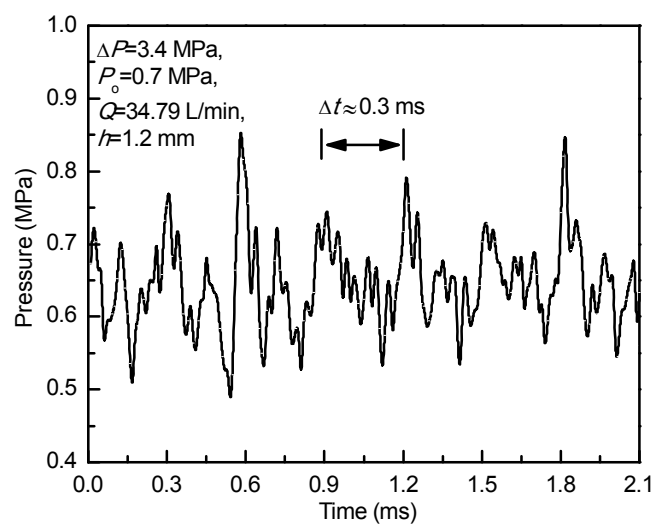

Fig. 2 Pressure fluctuation in the valve chamber after the generation of squeal noise

$\Delta P$ : pressure differential; $P_{\mathrm{o}}$ : outlet pressure; $Q$ : volumetric flow rate; $h$ : poppet lift

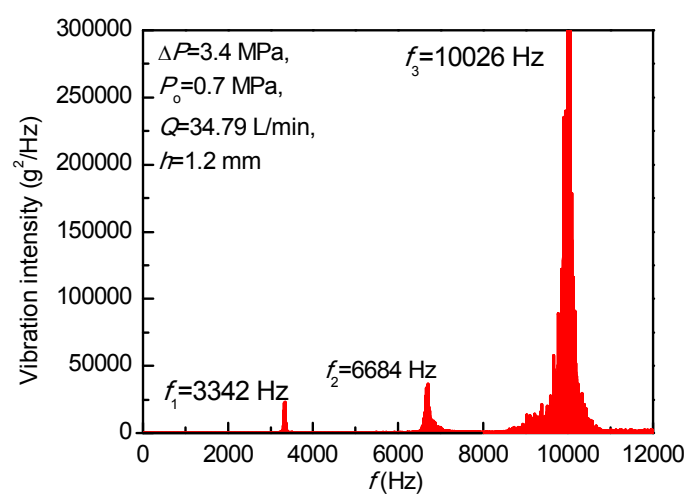

Fig. 3 Spectrum of squeal noise in test valve (V01)

\subsection{Excitation of the squeal noise in test valve}

Generally speaking, the poppet valve usually works at small openings and under high pressure drop conditions. On opening, the pressurized oil fluid issues from the narrow passage with high velocity and enters the sudden expansion cavity. The jet flow confined by the surrounding walls of valve body and poppet is naturally a submerged flow (Guo et al., 2001). When the jet flows into the expanded valve chamber, a recirculation zone is created in the presence of the valve chamber. The up-and-down movement of the recirculation cells nearby the submerged jet changes the local pressure and forces the jet flow to deflect to the low-pressure side, resulting in sustained oscillation with a feedback. This type of oscillatory flow is characterized by a low frequency (Lawson and Davidson, 1999). Moreover, the vortex shedding induced by the instability of the shear layer brings about a high-frequency oscillatory flow as well. The oscillation frequency of shear flow is usually illustrated by the Strouhal number, which is defined by Eq. (1). The characteristic length of the annular jet, as defined by Nakano et al. (1988), is the distance from the separation point of the wake to the impingement wall, which is denoted in Fig. 4.

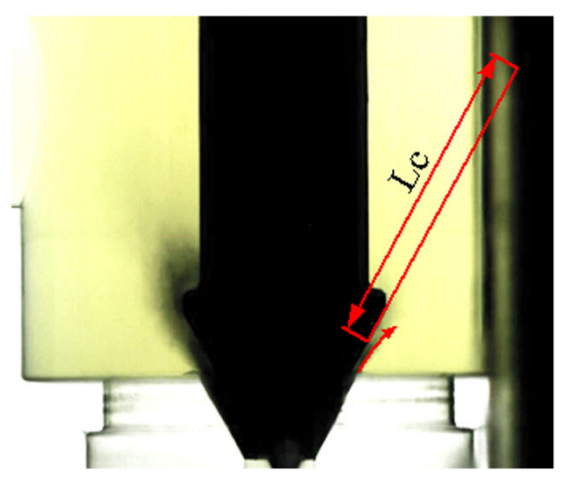

Fig. 4 Characteristic length of annular jet between separation point and the impingement

The oscillation of the shear flow in the fluid passage is highly related to the velocity of oil in the test valve. Fig. 5a shows the typical spectra of the sampling data when the test valve (V02) works under different flow velocity conditions. During the process of testing, the experiments were conducted by varying the inlet pressure at constant values of the valve opening and the backpressure. It is obvious that the fundamental frequency of squeal noise increases with an increase of flow velocity. The relation between the fundamental frequency of squeal noise and flow velocity is shown in Fig. 5b. The frequency of squeal noise increases linearly with flow 
velocity. The squeal noise may originate from the vortex shedding induced by the shear layer instability due to the high-frequency characteristics. Fig. 6 shows the Strouhal number of the test valve V02 when squeal noise is generated under various pressure and constant valve opening conditions. The Strouhal number ranges from 0.6 to 0.8 in our test, which is within the range $(0.5-2.5)$ for the shear layer instability phenomenon as measured by Rockwell and Naudascher (1979). An interesting phenomenon is that the Strouhal number is affected by the backpressure. As depicted in Fig. 6, the Strouhal number remains approximately a constant value when the valve V02 works at different pressure differential and constant backpressure conditions. While the Strouhal number increases as the backpressure increases, the pressure differential remains constant.

It is well known that cavitation is sensitive to the backpressure; thus, it is necessary to consider the effect of cavitation on the fundamental frequency of squeal noise.
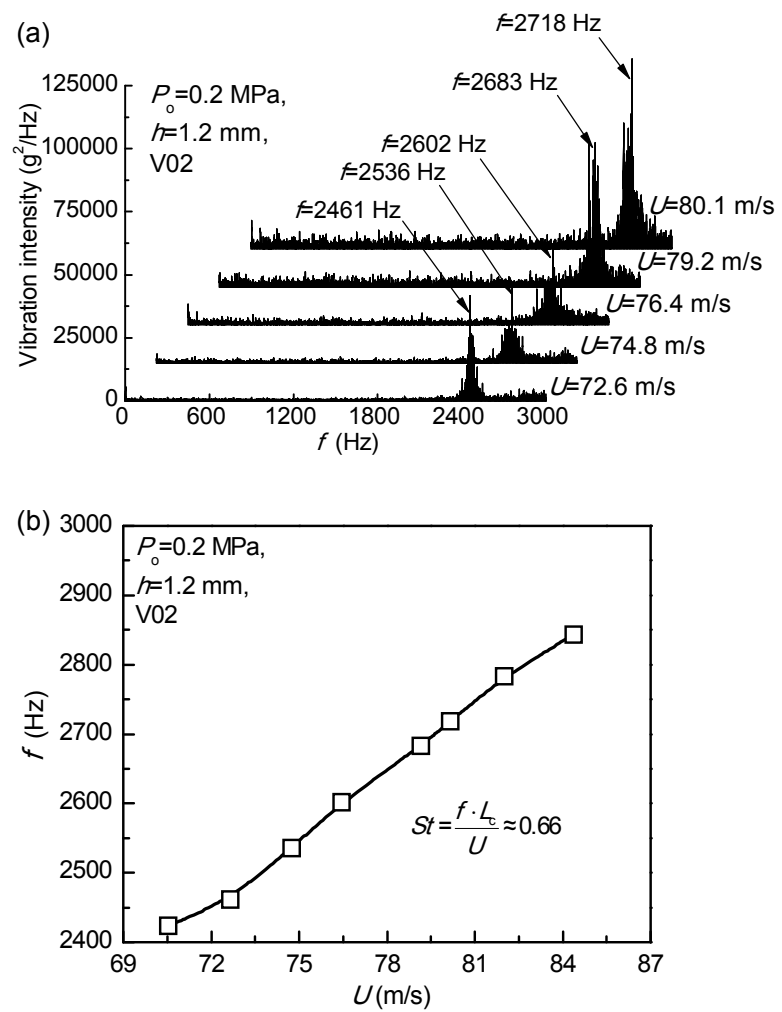

Fig. 5 Fundamental frequency of the squeal noise as a function of flow velocity $U$

(a) Spectral content of the squeal noise; (b) Frequency with flow velocity

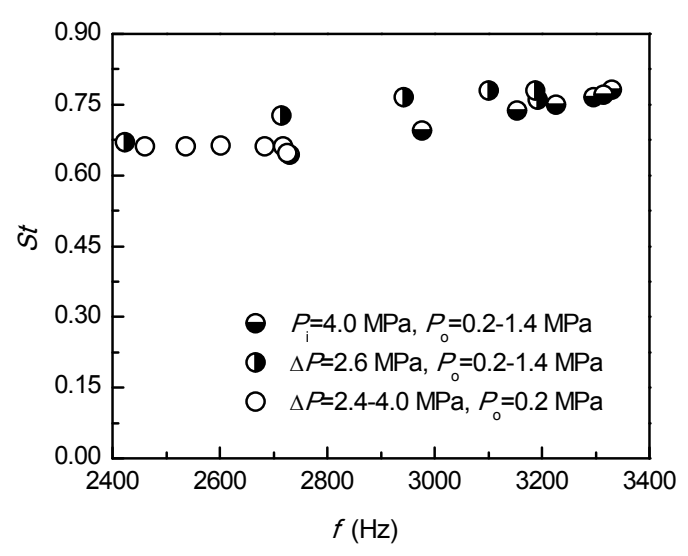

Fig. 6 Strouhal number versus fundamental frequency of the squeal noise (V02)

$P_{\mathrm{i}}$ : inlet pressure

\subsection{Helmholtz resonance in valve chamber}

An interesting phenomenon is that the fundamental frequency of the squeal noise is affected by not only the flow velocity, but also the valve's geometric parameters. Fig. 7 shows the tested fundamental frequency of the squeal noise generated in the valves V01 and V02, under the same conditions (with the same pressures and openings); both the valves have the same radial dimension but different axial dimensions of the valve chamber. The squeal noise generated in valve V01 with a small valve chamber has higher fundamental frequency compared with that of valve V02. These experimental phenomena have something in common with Helmholtz resonance.

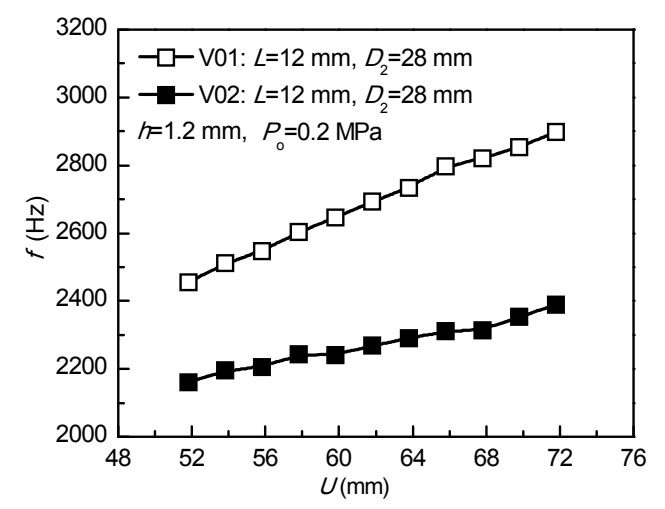

Fig. 7 Fundamental frequency of squeal noise generated in valves 
In the present test valve, the poppet with screwing rod is inserted in the valve chamber concentrically, forming an annular volume with an annular conical connector, with discontinuity of cross-sectional area at the interface of valve chamber and orifice, inducing multidimensional wave propagation in the volume (Dickey and Selamet, 1996). It is a challenge to deduce the analytic expressions of the resonant frequency for the poppet valve. It is fortunate that the classical expression for the resonant frequency of Helmholtz resonator is valid because the length dimension of the valve chamber is less than $1 / 16$ of the wavelength (Panton and Miller, 1975; Selamet and Lee, 2003). However, the classical expression for the resonant frequency of Helmholtz resonator is available for the resonator with a cylinder connector. Tang (2005) has given the resonant frequency expression of volume with tapered connector. The acoustic resonance frequency of the Helmholtz resonator with tapered connector is expressed as follows:

$$
f_{\text {res }}=\frac{C}{2 \pi} \sqrt{\frac{\pi r_{1} r_{2}}{H \times V}}=\frac{C}{2 \pi} \sqrt{\frac{S}{H \times V}},
$$

where $C$ is the sonic speed in fluid, $f_{\text {res }}$ is the acoustic resonance frequency of the resonator, $r_{1}$ and $r_{2}$ are the radii of the inlet and outlet cross-sections of the tapered connector, respectively, $S$ is the geometric mean section area of the tapered connector, and $V$ is the volume of the valve chamber.

In addition, the orifice shape is also a factor that affects the resonant frequency when the boundary layer thickness is comparable to the orifice size (Panton, 1990). But in this case, the boundary layer thickness, expressed as in Eq. (3) (Baker and Myhre, 1969), is much smaller than the orifice size. Therefore, the valve chamber can be simplified as a cylinder with a tapered connector, as shown in Fig. 8, where $S_{1}$ and $S_{2}$ are the sizes of the inlet and outlet cross-sections of the tapered connector, respectively.

$$
\delta=4.64 \sqrt{\frac{v x}{U}}
$$

where $\delta$ is the boundary layer thickness, $v$ is the kinematic viscosity of oil, and $x$ is the distance from the leading edge of the annular conical passage.
The calculated resonant frequencies of the resonators in valves V01 and V02 under the particular conditions of $h=1.3 \mathrm{~mm}, C=1380 \mathrm{~m} / \mathrm{s}$, and noncavitation are $4074 \mathrm{~Hz}$ and $3083 \mathrm{~Hz}$, respectively. In the process of the testing, it is found that squeal noise in the hydraulic poppet valve is usually generated in the case of cavitation occurrence. The maximum fundamental frequency of the squeal noise tested in valves V01 and V02 are $3809 \mathrm{~Hz}$ and $3300 \mathrm{~Hz}$ (cavitation), respectively, as shown in Fig. 9. Some errors $(<7 \%)$ appear between the experimental and the analytical results, and these would be caused by the simplification of the resonant frequency model and the effect of cavitation. However, the calculated results are very close to the experimental results.

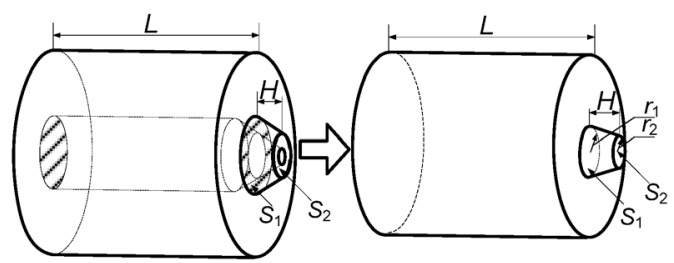

Fig. 8 Schematic diagram of the Helmholtz resonators

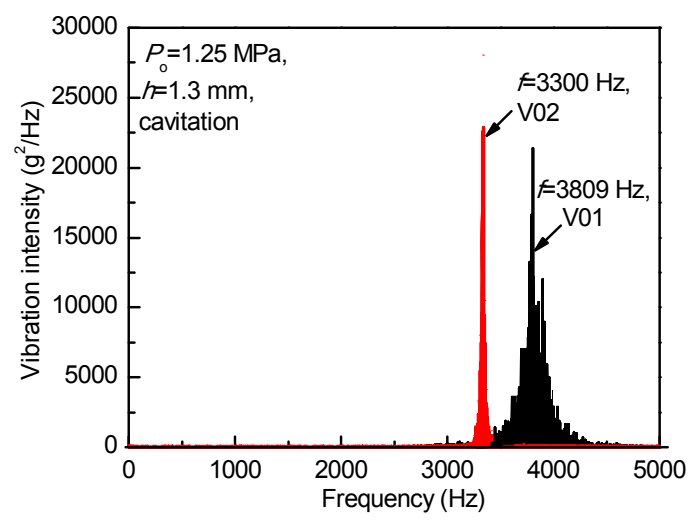

Fig. 9 Maximum fundamental frequency of squeal noise tested in valves under cavitation conditions

\subsection{Effects of fluid property}

Considering that squeal noise is caused by the Helmholtz resonance induced by the fluid-acoustic interaction, any alteration of factors related to the acoustic cavity's resonant frequency will affect the fundamental frequency of squeal noise. Fig. 10 shows the four groups of experimental results in terms of the fundamental frequency of squeal noise in valve V02 under different backpressures but 
constant valve opening conditions. It is found that the fundamental frequency of squeal noise is influenced considerably by the backpressure. As the valve operates under cavitation condition, the higher the backpressure is, the larger the fundamental frequency is. Once the backpressure increases and approaches the backpressure that can suppress the inception of cavitation, the fundamental frequency almost remains constant given an increase of backpressure. During the experiments, the identification of cavitation inception is important; cavitation inception is judged by visual and auditory methods, which are mentioned by Oshima and Ichikawa (1985). As backpressure decreases to a critical value at which a weak noise starts emanating and bubbles are observed in camera, cavitation inception is determined. The cavitation bubbles mixed in the oil can change the fluid's properties, such as density and bulk modulus of the fluid. The bubbles in the fluid decrease the bulk modulus significantly more than the density of the fluid, resulting in a reduction of the sonic speed $\left(C=(E / \rho)^{1 / 2}\right.$, where $E$ and $\rho$ are the bulk modulus and the density of fluid, respectively) (Kieffer, 1977). According to the expression on the resonant frequency of the Helmholtz resonator illustrated by Eq. (2), the sonic speed $C$ is proportional to the resonant frequency of the Helmholtz resonator. The higher the sonic speed is, the higher the resonant frequency becomes. The sonic speed is determined by the volume fraction of gas in the fluid, which is related to the extent of cavitation. For characterization of cavitation, the cavitation number $\sigma$ is introduced and defined as follows (Wang et al., 1998):

$$
\sigma=\frac{P_{\mathrm{o}}-P_{\mathrm{v}}}{0.5 \rho U^{2}}
$$

where $P_{\mathrm{v}}$ is the saturated vapor pressure of oil.

According to the expression of cavitation number, the cavitation number increases with the increase of backpressure at constant flow velocity. However, the greater the cavitation number is, the less the volume of vapor is (Amirante et al., 2014). Less bubbles mixed in the oil fluid results in the increase of sonic speed, causing an increase of resonant frequency. That is why the fundamental frequency increases as the backpressure increases.

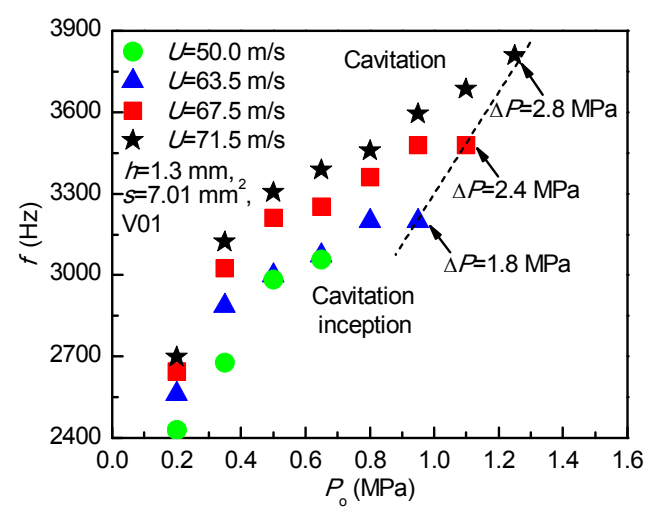

Fig. 10 Effects of backpressure on the fundamental frequency of the squeal noise

\subsection{Effects of the valve opening}

The acoustic impedance of the tapered connector, representing a measure of the amount of flow rate by which the motion induced by a pressure applied to a surface is impeded (Rienstra and Hirschberg, 2012), is another factor affecting the resonant frequency of the acoustic cavity. In the present experiments, the acoustic impedance is dependent on the throttling area at the neck of the conical passage, namely valve opening, which corresponds to the tapered connector's geometric mean section area $S$ listed in Eq. (2), and it increases with increase in poppet lift. Consequently, the larger valve opening implies the higher resonant frequency. Fig. 11a shows the fundamental frequency of squeal noise when the test poppet valve V02 operates at different poppet lifts. Experimental results are categorized into four groups based on flow velocity. The markers located at the horizontal axis mean no squeal noise is detected. Squeal noise occurs in the test valve V02 when the poppet lift increases to a certain value ( $h=1.0 \mathrm{~mm}$ in this case) in the cases of $U=76 \mathrm{~m} / \mathrm{s}$, $U=78 \mathrm{~m} / \mathrm{s}$, and $U=80 \mathrm{~m} / \mathrm{s}$, whereas in the case of $U=74 \mathrm{~m} / \mathrm{s}$, the occurrence of squeal noise is at a larger opening. When the acoustic resonant frequency of the Helmholtz cavity with a certain bandwidth overlaps partly with the excitation spectrum, the squeal noise is generated. As shown in Fig. 11b, after the occurrence of squeal noise, the fundamental frequency of the squeal noise has an increasing trend. The reason is because the acoustic resonant frequency of the Helmholtz resonator increases as the poppet lift increases. 

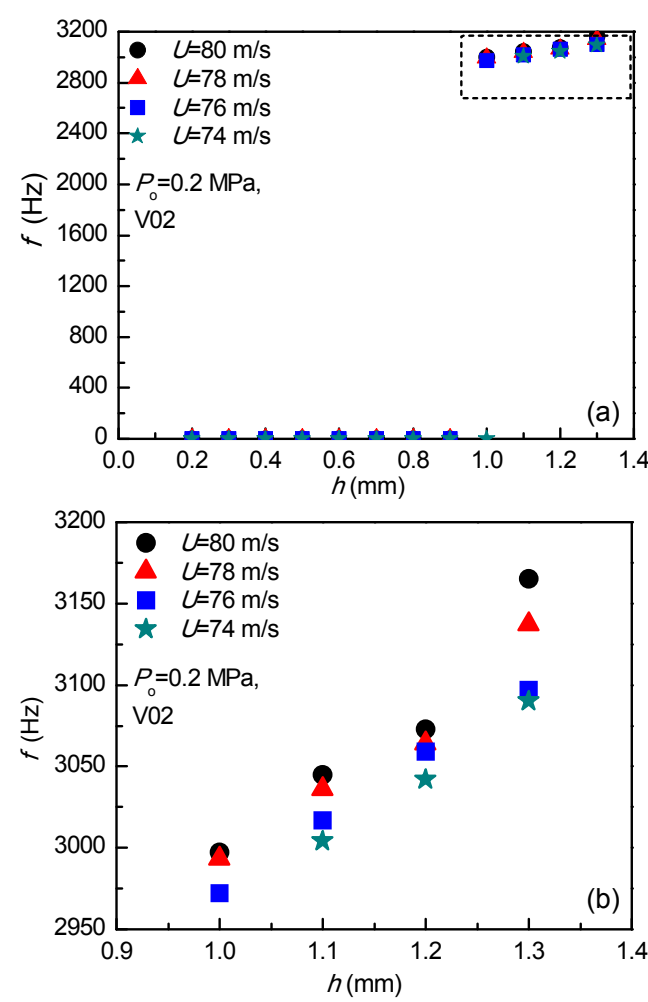

Fig. 11 Effects of valve opening on the fundamental frequency of squeal noise

(a) Fundamental frequency of squeal noise at various poppet lifts; (b) Amplified part marked with box in Fig. 11a

\section{Conclusions}

The squeal noise in poppet valves, which is characterized by a monotone tone and high frequency, is annoying. The suddenly expanded volume of the valve chamber at the exit of the narrow metering orifice forms a Helmholtz resonator. Actually, resonance is apt to occur if the external excitation frequency overlaps with that of the resonator; under this condition, a harsh squeal noise is emitted. The experiments show that a larger cavity volume, a smaller poppet lift, and a lower sonic speed in the fluid contribute to the decrease of squeal noise's fundamental frequency. It agrees well with the variation laws of the resonant frequency of an acoustic cavity. Moreover, the calculated resonant frequency of the poppet valve also verifies the hypothesis that the squeal noise is induced by the Helmholtz resonance. Thus, any factor that affects the acoustic resonant frequency of cavity influences the fundamental frequency of the squeal noise as well. Furthermore, the squeal noise can be suppressed effectively by removing the frequency overlaps between the resonant frequency of the valve chamber and the exciting frequency induced by shear layer instability. The exciting frequency of fluid is highly related to the flow velocity; actually, it is not possible to alter the exciting frequency induced by shear layer instability without significant loss of the flow capacity. By contrast, the modification of the valve chamber's resonant frequency can be achieved easily given that valve chamber space constraint exists. The resonant frequency of the poppet valve chamber can be changed considerably by modifying the length or the crosssectional area size of the conical passage.

\section{References}

Amirante, R., Distaso, E., Tamburrano, P., 2014. Experimental and numerical analysis of cavitation in hydraulic proportional directional valves. Energy Conversion and Management, 87:208-219.

http://dx.doi.org/10.1016/j.enconman.2014.07.031

Baines, P.G., Mitsudera, H., 1994. On the mechanism of shear flow instabilities. Journal of Fluid Mechanics, 276:327-342. http://dx.doi.org/10.1017/S0022112094002582

Baker, D.N., Myhre, D.L., 1969. Effects of leaf shape and boundary layer thickness on photosynthesis in cotton (Gossypium hirsutum). Physiologia Plantarum, 22(5): 1043-1049. http://dx.doi.org/10.1111/j.1399-3054.1969.tb07463.x

Balatka, K., Mochizuki, S., Murata, A., 1996. Flow in an annular-conical passage. JSME International Journal Series B, Fluids and Thermal Engineering, 39(1):66-71. http://dx.doi.org/10.1299/jsmeb.39.66

Dickey, N.S., Selamet, A., 1996. Helmholtz resonators: onedimensional limit for small cavity length-to-diameter ratios. Journal of Sound and Vibration, 195(3):512-517. http://dx.doi.org/10.1006/jsvi.1996.0440

Guo, B., Langrish, T.A.G., Fletcher, D.F., 2001. An assessment of turbulence models applied to the simulation of a two-dimensional submerged jet. Applied Mathematical Modeling, 25(8):535-653. http://dx.doi.org/10.1016/S0307-904X(00)00068-8

Kieffer, S.W., 1977. Sound speed in liquid-gas mixtures: water-air and water-steam. Journal of Geophysical Research, 82(20):2895-2904. http://dx.doi.org/10.1029/JB082i020p02895

Lawson, N.J., Davidson, M.R., 1999. Crossflow characteristics of an oscillation jet in a thin slab casting mould. Journal of Fluids Engineering, 121(3):588-595. http://dx.doi.org/10.1115/1.2823509

Lu, L., Zou, J., Fu, X., 2012. The acoustics of cavitation in spool valve with U-notches. Proceedings of the 
Institution of Mechanical Engineers, Part G: Journal of Aerospace Engineering, 226(5):540-549. http://dx.doi.org/10.1177/0954410011413221

Nagaya, Y., Murase, M., 2012. Detection of cavitation with directional microphones placed outside piping. Nuclear Engineering and Design, 249:140-145. http://dx.doi.org/10.1016/j.nucengdes.2011.08.045

Nakano, M., Outa, E., Tajima, K., 1988. Noise and vibration related to the patterns of supersonic annular flow in a pressure reducing gas valve. Journal of Fluids Engineering, 110(1):55-61. http://dx.doi.org/10.1115/1.3243511

Oshima, S., Ichikawa, T., 1985. Cavitation phenomena and performance of oil hydraulic poppet valve: 1st report. Mechanism of generation of cavitation and flow performance. Transactions of the Japan Society of Mechanical Engineers Series B, 51(462):427-435. http://dx.doi.org/10.1299/kikaib.51.427

Panton, R.L., 1990. Effect of orifice geometry on Helmholtz resonator excitation by grazing flow. AIAA Journal, 28(1):60-65. http://dx.doi.org/10.2514/3.10353

Panton, R.L., Miller, J.M., 1975. Resonant frequencies of cylindrical Helmholtz resonators. The Journal of the Acoustical Society of America, 57(6):1533-1535. http://dx.doi.org/10.1121/1.380596

Porteiro, J.L.F., Weber, S.T., Rahman, M.M., 1997. An experimental study of flow induced noise in counterbalance valves. International Symposium on FluidStructure Interactions, Aeroelasticity, Flow-Induced Vibration and Noise, Dallas, USA, p.557-562.

Rahman, M.M., Porteiro, J.L.F., Weber, S.T., 1997. Numerical simulation and animation of oscillating turbulent flow in a counterbalance valve. Energy Conversion Engineering Conference, IECEC-97, Proceedings of the 32nd Intersociety, Honolulu, USA, p.1525-1530. http://dx.doi.org/10.1109/IECEC.1997.661996

Raman, G., 1999. Supersonic jet screech: half-century from Powell to the present. Journal of Sound and Vibration, 225(3):543-571. http://dx.doi.org/10.1006/jsvi.1999.2181

Rienstra, S.W., Hirschberg, A., 2012. An Introduction to Acoustics. Eindhoven University of Technology, Eindhoven, p.36-37.

Rockwell, D., Naudascher, E., 1979. Self-sustained oscillations of impinging free shear layers. Annual Review of Fluid Mechanics, 11(1):67-94. http://dx.doi.org/10.1146/annurev.fl.11.010179.000435

Selamet, A., Lee, I., 2003. Helmholtz resonator with extended neck. The Journal of the Acoustical Society of America, 113(4):1975-1985. http://dx.doi.org/10.1121/1.1558379

Shin, Y.C., 1991. Static and dynamic characteristics of a two stage pilot relief valve. Journal of Dynamic Systems,
Measurement, and Control, 113(2):280-288.

http://dx.doi.org/10.1115/1.2896376

Tang, S.K., 2005. On Helmholtz resonators with tapered necks. Journal of Sound and Vibration, 279(3-5):10851096. http://dx.doi.org/10.1016/j.jsv.2003.11.032

Testud, P., Aurégan, Y., Moussou, P., et al., 2009. The whistling potentiality of an orifice in a confined flow using an energetic criterion. Journal of Sound and Vibration, 325(4-5):769-780. http://dx.doi.org/10.1016/j.jsv.2009.03.046

Wang, Y.G., Shintani, M., Liu, S.J., et al., 1998. Cavitation characteristics around a hollow jet valve (observation by high-speed photographs and monitoring by vibration). Japanese Turbomachinery Society, 26(1998):361-368. http://dx.doi.org/10.11458/tsj1973.26.361

\section{中文概要}

题 目: 液压雉阀中的啸叫噪声

目的: 液压锥阀产生尖锐刺耳的啸叫噪声, 严重地降 低了液压锥阀的品质。本文探讨液压锥阀啸叫 噪声的产生机理, 揭示啸叫噪声对特定频率噪 声信号的选择性放大原理, 为啸叫噪声抑制提 供理论依据。

创新点: 观测到介质相变、雉阀开度及阀腔变化下啸叫 噪声基频漂移现象, 建立空化条件下雉阀流体 共振分析模型, 获得了雉阀啸叫噪声是对特定 频率信号的选择性放大的结论。

方 法: 1. 通过实验分析, 观测到液压雉阀中啸叫噪声 基频的漂移规律（图 5 7、10 和 11）；2. 提出 液压锥阀啸叫噪声是流声耦合引起的亥姆霍兹 共振假设; 3. 通过建立雉阀阀腔声学共振频率 模型, 运用实验与理论相结合的方法分析不同 工况下的啸叫噪声基频漂移规律, 通过大量实 验验证所提假设的正确性（图 7、10 和 11）。

结 论: 1. 液压锥阀流声共振产生啸叫噪声, 完成对特定 频率噪声信号的选择性放大; 2. 液压锥阀只有在 流体不稳定产生的周期性压力扰动信号的频率与 阀腔声学共振频率接近时才产生啸叫噪声; 3. 运 用液压雉阀流声共振产生啸叫噪声的结论, 通过 改变阀腔声学共振频率, 使之与流道内流体不稳 定引起的压力扰动频率错开, 能有效地抑制液压 雉阀中啸叫噪声的产生。

关键词: 雉阀; 啸叫噪声; 亥姆霍兹共振; 流声共振; 剪切层不稳定 